\title{
Electrolyte Absorption Alteration
}

National Cancer Institute

\section{Source}

National Cancer Institute. Electrolyte Absorption Alteration. NCI Thesaurus. Code

C41551.

.A finding indicating a deficiency or excess in biological absorption or assimilation of one or more electrolytes. 